\title{
Low-cost CDC ROADM Architecture Based on Stacked Wavelength Selective Switches
}

\author{
Haining Yang, Brian Robertson, Peter Wilkinson, and Daping Chu
}

\begin{abstract}
A highly flexible, stacked, switch module is proposed, wherein multiple independent $1 \times N$ wavelength selective switches (WSSs) can be realised on a single $4 \mathrm{k}$ liquid crystal on silicon (LCOS) device. The stacked WSSs module can be configured in different ways for application at either the transit side or the add/drop side of a colourless, directionless and contentionless (CDC) reconfigurable optical add/drop multiplexer (ROADM). Two ROADM architectures are proposed based on the stacked WSSs modules. Their costs are analysed for both a 4-degree network node and a larger 8-degree node. The first proposed ROADM architecture with full CDC features is shown to realise a cost reduction of at least $35 \%$ in these two test network nodes, when compared with the conventional CDC ROADM architecture based on the standalone WSSs and multicasting switches (MCSs). The second ROADM architecture proposed has a small probability of wavelength contention, which could be prevented by a local wavelength assignment algorithm. According to our cost estimation, it is able to aggressively reduce the number of components at the add/drop side, and make an overall cost reduction of $>70 \%$ and $>80 \%$ in the 4-degree and 8-degree network nodes, respectively.
\end{abstract}

Index Terms - Liquid crystal on silicon; reconfigurable optical add/drop multiplexer; wavelength selective switch.

\section{INTRODUCTION}

$\mathbf{R}$ configurable Multiplexers (ROADMs) [1,2] have become the key building block for modern wavelength division
multiplexing (WDM) optical networks. ROADMs are able to redirect the optical signals from any incoming direction of a network node to any of its outgoing directions on a

Manuscript received November 28, 2016.

Haining Yang, Brian Robertson, Peter Wilkinson and Daping $\mathrm{Chu}^{*}$ are with Roadmap Systems Ltd, St John's Innovation Centre, Cambridge CB4 0WS, UK. (“daping.chu@roadmapsystems.co.uk).

Peter Wilkinson and Daping Chu are also with Centre for Photonic Devices and Sensors, Department of Engineering, University of Cambridge, 9 JJ Thomson Avenue, Cambridge CB3 0FA, UK. per-wavelength basis, without the need for optical-electrical-optical (OEO) conversion. ROADMs with colourless, directionless and contentionless (CDC) features also allow service providers to drop any wavelength channel from any direction to any local transceivers or add any wavelength channel from a local transceiver to any outgoing direction. Therefore, CDC ROADMs promise simplified network management and reduced operating expense.

The key enabling technology for ROADMs is the wavelength selective switch (WSS) [3,4]. A typical WSS has one input fibre port and $N$ output fibre ports. It can selectively route individual WDM input channels to any of the output fibre ports, according to the software configuration which is remotely controlled by the service providers. WSSs are widely used in ROADMs to redirect the wavelength channels from one degree to another.

In recent years, phase-only liquid crystal on silicon (LCOS) spatial light modulators (SLMs) [5] have become the technology of choice for WSSs, due to their software upgradable nature and support for flexible spectrum switching [6]. Efforts have also been made to improve the port count [7-9], passband shape [10], and static [11-15] and transient [16,17] crosstalk levels in LCOS WSSs. WSSs are usually based on the 'disperse-and-select' optical design [18], where the WDM channels from the input port are diffracted along the dispersion axis at the LCOS plane, before being switched to the target output ports by the sub-holograms displayed on the corresponding areas of the LCOS device. Due to the limited number of pixels available on the current generation of LCOS devices, anamorphic optics are invariably used to convert the input signals to elongated beams at the LCOS plane. The output ports are correspondingly arranged along a switching axis that is orthogonal to the dispersion axis. Although such an approach is able to increase the port count in one axis, it fails to fully exploit the two-dimensional (2D) nature of the LCOS pixel array. Moreover, for such a configuration, all the undesirable diffraction orders from LCOS quantization effects will also appear along this switching axis, which makes it fundamentally difficult to suppress crosstalk, especially in WSSs with high port counts. In addition, the optical system for the WSS requires high alignment accuracy, which increases the unit cost [19]. We have also demonstrated that the LCOS device itself can be used to give alignment feedback [20].

The add/drop side of the CDC ROADMs can employ $M \times N$ multicasting switches [20], which pairs an array of 
$1 \times N$ splitters with another array of $1 \times M$ space switches (SS). Multicasting switches have high insertion loss, which would further increase as the ROADM node is expanded. Therefore, it is fundamentally difficult to scale. The insertion loss and the scalability issue could be resolved by replacing the splitter array with a WSS array [21]. However, the relatively high unit cost and footprint of current-generation WSSs make this approach unattractive. Recently, a new architecture based on the contentionless $M \times N$ WSS [22-24] was proposed as a way of reducing the overall cost of the ROADM, although the $M \times N$ WSS used is complicated to construct and therefore is also costly Therefore, it is still unclear whether the benefits brought by CDC features can justify the extra complexity and cost of these CDC add/drop implementations.

In this paper, we propose low-cost CDC ROADM architectures based on a stacked WSSs design [25]. Since the stacked WSS design does not use anamorphic optics, it can utilise $2 \mathrm{D}$ beam steering and incorporate a large number of flexible spectrum $1 \times N$ WSSs on a single $4 \mathrm{k}$ LCOS device. These WSSs would share a large portion of their optical components, and have a common alignment procedure, leading to a lower unit cost and smaller footprint. This makes it economically viable to deploy large numbers of WSSs in a network node. This work details how to use the stacked WSSs module in the transit and add/drop side of a CDC ROADM and describes the benefits it will bring. The costs of these implementations are analysed and compared with conventional approaches based on multicasting switches and standalone WSS modules.

\section{Stacked WSSs Design AND Cost Estimation}

Fig. 1 shows the design principle of the $M$ stacked $1 \times N$ WSSs, which consists of an array of objective lenses $\left(\mathrm{L}_{\mathrm{A}}\right)$, a relay system $\left(\mathrm{L}_{1}\right.$ and $\left.\mathrm{L}_{2}\right)$, DEMUX optics $\left(\mathrm{P}_{\mathrm{g}}\right)$ and an LCOS device. In this example, 3 independent $1 \times 8 \mathrm{WSSs}$ are stacked. Each WSS in this stacked design has a fibre array cluster, which consists of 1 input fibre port, $N$ output fibre ports, and a corresponding micro-lens array. These clusters are arranged along the y-axis, each acting as an independent WSS, with each input light beam illuminating a spatially distinct row of sub-holograms (e.g., $\mathrm{S}_{1}, \mathrm{~S}_{2}$, and $\mathrm{S}_{3}$ ). The input WDM channels are launched into each WSS via the central fibre of the corresponding cluster. The objective lens generates a beam waist of radius $\omega_{0}$ at plane $\mathrm{P}_{\mathrm{o}}$. The $4 \mathrm{f}$ relay system images this beam waist at the SLM plane (PSLM). Between the relay lenses, a static grating $\left(\mathrm{P}_{\mathrm{g}}\right)$ imparts an angular displacement of $B_{G}(\lambda)$ to each wavelength channel in the $x-z$ plane. Signal beams associated with these wavelengths illuminate separate sub-holograms displayed on the LCOS device. The sub-hologram for a wavelength channel could be a grating of period T, orientated at an angle of $\varphi$ with respect to the local $x y$-coordinate system, that diffracts the light beam such that it leaves the LCOS SLM with a propagation vector of $\mathrm{k}(\rho, \varphi, \lambda)$, where $\rho$ is the angle of the vector with respect to the local z-axis. The diffracted beam is subsequently imaged at $\mathrm{P}_{\mathrm{o}}$ by the relay system. The objective lens in the output optics shown in Fig. 2 converts the propagation vector of a wavelength channel, $k(\rho, \varphi, \lambda)$, to

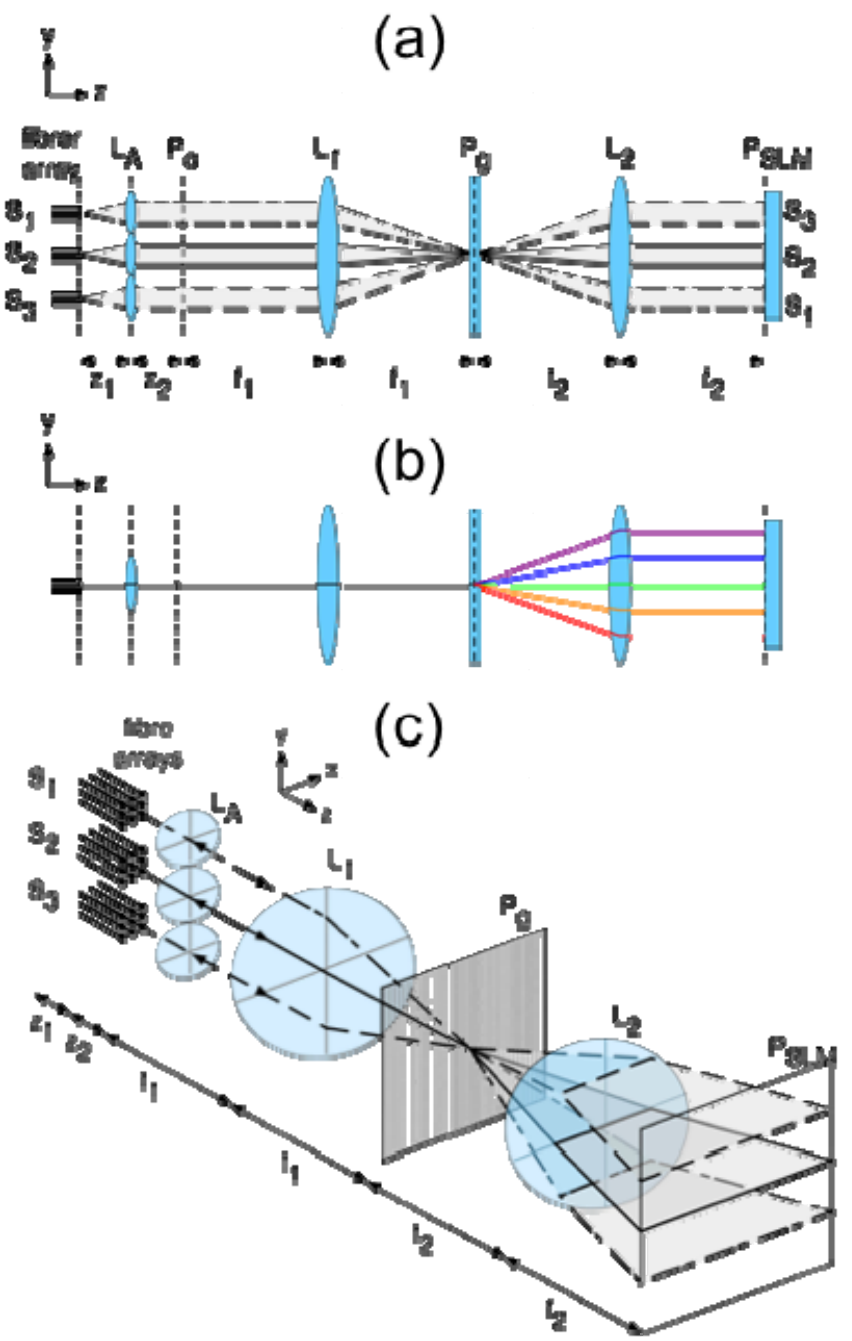

Fig. 1 Principle of a stacked WSSs based on a single LCOS device, (a) side view, (b) top view and (c) system view.

a beam position that is offset from the optical axis. The angle is controlled such that the beam is concentric with respect to the intended output fibre, thereby maximising coupling efficiency, as shown in Fig. 2(a). A secondary lenslet array, $L_{F}$, focuses the wavelength channels into the output fibre array.

Without using anamorphic optics, the number of pixels required along the y-axis of the LCOS SLM is significantly reduced. A large number of independent WSSs can therefore be stacked together to fully exploit the pixel count of the LCOS device and share the common optics, i.e. the relay optics and the diffraction grating. Our previous work [25] has shown that it is possible to achieve high-accuracy beam steering by only allocating $50 \times 50$ pixels on the LCOS device for each $50 \mathrm{GHz}$ channel within the C-band. By using a standard 4k LCOS device (such as the Jasper JD2704 with $4096 \times 2400$ pixels), the whole $\mathrm{C}$-band can be covered. If a polarisation-insensitive LCOS device [26] is used, 48 independent WSSs can be stacked along the y-axis of a single chip in total. This stacked WSS architecture is also compatible with the polarisation-diversity implementation proposed in [27], where the beams from the two polarisations 

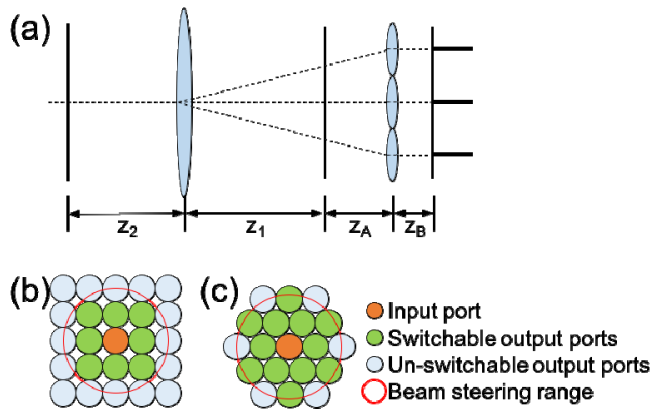

Fig. 2. (a) Output optics design; $2 \mathrm{D}$ beam steering over the fibre ports arranged on (b) a Cartesian grid and (c) a hexagonal grid.

can share a same area on the LCOS device. Therefore, the number of WSSs that can be stacked along the y-axis does not need to halved for the polarisation insensitive operation.

In the absence of anamorphic optics, the un-modulated input signal to each sub-hologram has a circular beam shape on PSLM, which is designed to cover $31 \times 31$ pixels, and hence achieve the $4^{\text {th }}$ order super Gaussian passband shape. The small number of pixels covered by the beam limits the maximum grating period to 10 pixels, which is larger than the minimum period of 7 pixels required to realise sufficient switching efficiency and reasonably low crosstalk level for two switchable positions along a given axis. The circular beam on PsLm allows 2D steering, giving 8 switchable output ports arranged on a Cartesian grid, as shown in Fig. 2(b). The number of the switchable output ports can be further increased, given the same beam steering range, to 12 if the fibre ports are arranged in a hexagonal pattern, as shown in Fig. 2(c).

It should be noted that the port count in each stacked WSSs can be further increased by using anamorphic optics to elongate the beams along the y-axis on PsLm so that the number of switchable positions is increased along this axis, though the number of WSSs incorporated on an LCOS device will be reduced. It is realistic to expect to stack 8 independent $1 \times 32$ WSSs in this way.

Efforts have also been made to estimate the cost of the stacked WSSs module, which is still under development. According to [28], the cost estimation of a WSS system was carried out based on the assembly and packaging complexity and the component costs. It is assumed that the assembly and packaging contribute to $40 \%$ of the cost of a standalone $1 \times \mathrm{N}$ WSS while the remainder is due to the components. The component costs are primarily driven by the costs of the thermally-stabilised phase-only LCOS device and the bulk optics, including the diffraction grating and relay lenses. Table I lists the estimated cost breakdown for individual components within a standalone $1 \times \mathrm{N}$ WSS. These values will be used as the reference in the following analysis of the overall cost of a stacked WSSs module.

When compared with a conventional standalone $1 \times \mathrm{N}$ WSS, the component count in a stacked WSSs system remains the same, although the physical dimension of certain components may need to be increased, to accommodate the larger number of WSSs. This implies that the stacked WSSs architecture will not necessarily introduce extra assembly steps. For example, the stacked WSSs module will only require a single fibre array and one
TABLE I

Cost BREAKDOWn OF THE STANDALONE $1 \times$ N WSSs AND THE STACKED WSSS MODULE

\begin{tabular}{lccc}
\hline \hline & Standalone & $\begin{array}{c}\text { Stacked } \\
\text { (std.) }\end{array}$ & $\begin{array}{c}\text { Stacked } \\
\text { (cons.) }\end{array}$ \\
\hline Assembly \& packaging cost & 0.40 & 0.40 & 0.50 \\
\cline { 2 - 4 } LCOS device & 0.20 & 0.20 & 0.20 \\
Diffraction grating & 0.20 & 0.60 & 0.70 \\
Relay lens & 0.15 & 0.20 & 0.30 \\
Fibre array cluster & 0.05 & 0.10 & 0.15 \\
\hline Total & 1.00 & 1.50 & 1.85 \\
\hline \hline
\end{tabular}

matched collimating micro-lens array. Individual WSSs in the module would share different sections of this clustered fibre array, as illustrated in Fig. 3. Therefore, the required number of assembly steps is not linearly dependent on the number of stacked WSSs in the module. Moreover, the stacked WSS architecture does not impose more stringent alignment requirements with regard to the optical components, especially the fibre array cluster, which normally would have the tightest assembly tolerances. Therefore, in principle, a conventional assembly procedure can be employed during the construction of the stacked WSS module. Based on these factors, authors estimate that the total assembly and packaging costs for the stacked WSS module would be similar to, or only slightly higher than, the assembly cost associated with a standalone $1 \times \mathrm{N}$ WSS module.

We also compared the complexity and dimensions of individual components in the stacked WSS module with their counterparts in a standalone $1 \times \mathrm{N}$ WSS module. Firstly, the overall cost of the LCOS device and its temperature stabiliser is not expected to increase, considering all the stacked WSSs share the same LCOS device. The physical dimensions of the diffraction grating, however, is likely to increase substantially, to accommodate the relatively larger incident beams at its plane. Consequently, it is assumed that its cost could be tripled based on the relationship between size and cost [29]. Depending on the number of stacked switches, optimisation of the relay lenses may be needed to

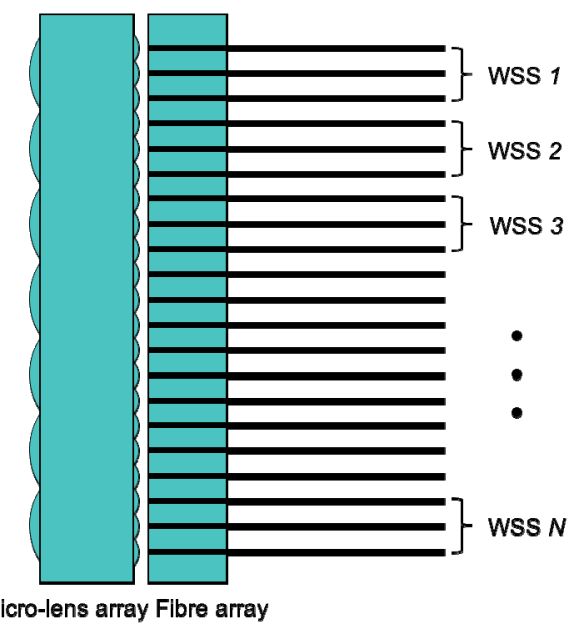

Fig. 3. Fibre array cluster assembly based on a fibre array and a micro-lens array for all the stacked WSSs. 
minimise the aberrations such as field curvature, which will have cost implications. However, it has also been recently demonstrated [30] that the aberrations in a WSS system can be compensated for by displaying a specially designed beam steering phase pattern on the LCOS device without incurring extra costs. The number of fibres and the corresponding micro-lenses need to be significantly increased. However, the polishing process contributes to a significant part of the fibre array cost. It is incurred on the per array basis instead of per fibre basis. Meanwhile, micro-lens arrays are manufactured on the wafer basis, which normally promises good scalability. Therefore, we believe that the costs of both components will scale favourably against the fibre count.

Based on this analysis, the estimated cost breakdown of the stacked WSS module at the standard component level is given in Table I, with the reference to that of a standalone WSS module. As the overall costs are sensitive to the cost increase of individual components, a more conservative estimation with higher costs is also given in the same table. By assuming the cost uncertainty of each component is the same for all different WSS configurations, the cost sensitivity and uncertainty of the stacked WSS module can be established by using Table I. This will in turn help to establish the cost sensitivity of the CDC ROADM architectures to the cost of the stacked WSS module. In both cost scenarios for the stacked WSS, the extensive sharing of the components and alignment costs between WSSs in the stacked WSS module significantly reduces the cost, both per WSS and per port.

\section{STACKED WSSS FOR ROADM TRANSIT}

The stacked WSS architecture significantly reduces the cost and footprint per WSS and per port. Therefore, it opens the possibility of implementing cost effective ROADM architectures that employ a large number of WSSs. This section will detail how a CDC ROADM can benefit from the stacked WSS module.

Fig. 4 shows the transit side of a ROADM based on the 'route-and-select' architecture, which interfaces the local add/drop side. The transit side of a ROADM is responsible for re-directing the WDM channels from one direction to another. In this architecture, the incoming WDM channels from a network direction will pass through a couple of paired $1 \times N$ WSSs. The first $1 \times N$ WSS routes the signals to the destined direction, while the second multiplexes the signals together with those from other directions for further transmission. Although it is possible to replace the $1 \times N$ WSS (used in reverse) at the exit side of each direction with a $1 \times N$ coupler to realise the same routing functions, the $1 \times N$ WSSs provide better channel isolation and lower insertion loss, especially in a multi-degree ROADM.

For an $R$-degree ROADM based on this architecture, each direction requires $21 \times N \mathrm{WSSs}$, and therefore $2 \mathrm{R} 1 \times N \mathrm{WSSs}$ are required in total. For each $1 \times N$ WSS, the port count $\mathrm{N}$ need to be at least $R$, i.e. $(\mathrm{R}-1)$ ports to be connected with WSSs for other directions while at least one port is reserved for the add/drop interfaces. For the example of a 4-degree ROADM node shown in Fig. 4, $81 \times N$ WSSs are required, where $\mathrm{N}$ needs to be at least 4 . Moreover, it is common in the modern networks that more than one fibre is bundled

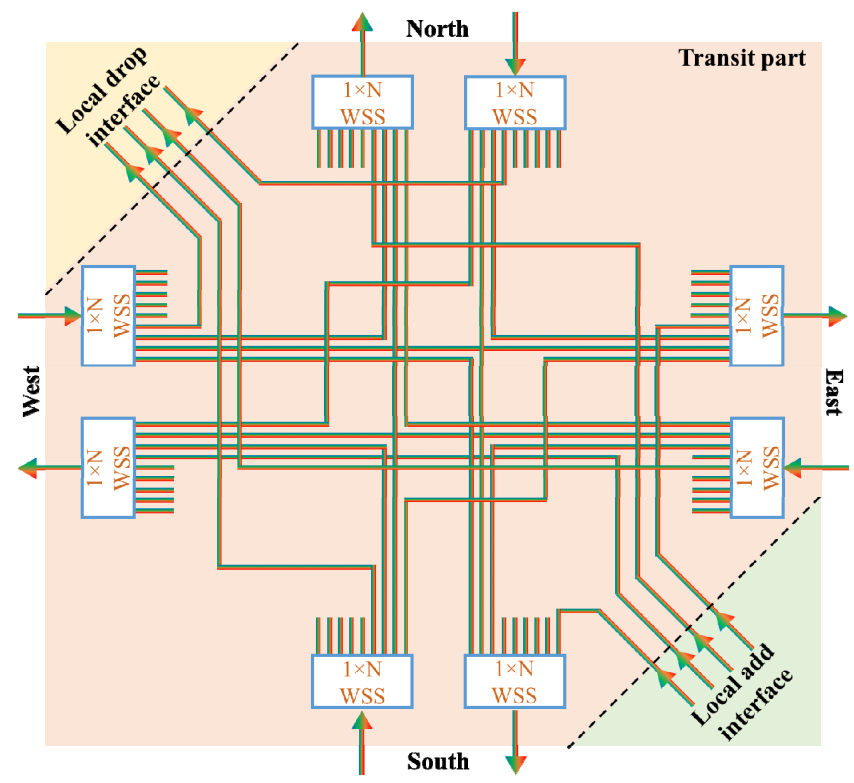

Fig. 4 The transit side of a 4-degree ROADM based on the 'route-and-select' architecture.

together to link two network nodes. In other words, the network node needs to be able to interface the parallel fibres from the same direction. Normally, multiple ROADMs are needed to handle these fibres.

The stacked WSS module proposed in the previous section could be used here to replace all the standalone WSSs within a single ROADM. This highly-integrated solution will be able to realise the 'route-and-select' switching features at lower cost and footprint. In this case, however, the stacked WSS module would also become a single point of failure. If the LCOS device for the stacked WSS module failed, the fibres for all the directions that are connected to this stacked WSS module would become unavailable. Although the protection scheme in $[22,23]$ could be put into place to avoid this situation, the extra components required would increase the complexity and cost.

Instead of using a stacked WSS module to handle all the wavelength-level routing within a single ROADM, we propose to use the stacked WSSs to interface with the parallel fibres entering or exiting a network node from the same direction. The parallel fibres are normally bundled together and therefore potentially cause a single point of failure issue as well. Network operators have already had protection schemes in place to avoid the loss of traffic. As a result, the stacked WSS module, when being used to handle such parallel fibres entering or exiting a network node, will not introduce extra risk of failure from the perspective of the whole network system. We therefore believe that such an approach is a more sensible way to exploit the benefit of a stacked WSS module.

Note that in some application scenarios, the ability to route wavelengths from any fibre pair, to any fibre pair, is provided within today's deployed multi-degree ROADMs by using an independent degree assigned to each fibre pair. In that case the number of degrees in the ROADM is effectively multiplied by the number of fibre pairs. This would require the individual WSS at the transit side has a high port count. As long as the stacked WSSs have high enough port count, 
there should be no problem to support such a multi-degree ROADM architecture.

\section{STACKED WSSs FOR CDC AdD/DROP IN ROADM}

The current commercially available $\mathrm{CDC}$ add/drop solution for ROADM is based on the $M \times N$ multicasting switch, in which an array of $M 1 \times N$ splitters/couplers are paired with another array of $N 1 \times M$ space switches. In the example shown in Fig. 5, $M=4$ and $N=16 . M \times N$ multicasting switches are designed for an $M$-degree network node and are able to add $N$ wavelength channels to the network node in a $\mathrm{CDC}$ fashion or vice versa. In this architecture, the insertion loss from the $1 \times N$ splitters/couplers alone will be at least $-10 \log _{10}(1 / N)$. Even for a few-degree network node, where the number of wavelength channels that need to be added or dropped is low, an array of amplifiers is needed to compensate for the loss and maintain a good optical signal to noise ratio (OSNR). Tunable filters would also be required by the transceivers at the drop side shown in Fig. 5(b) if the transceivers do not have coherent detection capability, as the multicasting switch itself does not have a filtering function. Given the high cost of tunable filters, especially those compatible with the flexible spectrum standard, it would only be cost effective to deploy CDC add/drop solutions based on the multicasting switches in conjunction with the transceivers with the coherent detection capability.

In a typical node each input fibre carries 80 wavelength channels, and $20 \%$ of them are dropped for local processing. As a result, $\mathrm{N}_{\text {drop }} M \times N$ multicasting switches are required for the CDC drop operation in a $R$-degree ROADM node, where $N_{\text {drop }}=R \times 80 \times 0.20 / N$. Accordingly, the port count of the $1 \times N$ WSSs used in the transit side will be $R+N_{\text {drop }}-1$. Considering the same amount of channels is also need to be added to the network node for further transmission, the equivalent number of $M \times N$ multicasting switches will be required for the $\mathrm{CDC}$ add operation. Due to the high loss of the $M \times N$ multicasting switches, an array of $M$ amplifiers need to be placed at the interface between each multicasting switch and the transit side of the ROADM. In total, $2 \mathrm{M} \times \mathrm{N}_{\text {drop }}$ amplifiers are required for the CDC add/drop operation, increasing CAPEX and OPEX for the node.

As shown in Fig. 6, the scalability issue associated with the $M \times N$ multicasting switches can be resolved by replacing splitters/couplers with an array of $1 \times N$ WSSs, since the insertion loss of $1 \times N$ WSSs does not scale with the port count as it does for $1 \times N$ splitters/couplers. When the $1 \times N$ WSSs and $1 \times M$ space switches are paired in this way, they constitute a contentionless $M \times N$ wavelength cross-connect (WXC), which is a CDC add/drop solution. Such WXCs will typically have an insertion loss of $7 \mathrm{~dB}$, i.e. $6 \mathrm{~dB}$ from the WSSs and $1 \mathrm{~dB}$ from the space switches. On top of the $6 \mathrm{~dB}$ insertion loss of the WSSs in the transit side of the ROADM, a total loss of $13 \mathrm{~dB}$ is expected for the added or dropped wavelength channels. This is still well within the typical power budget of $18 \mathrm{~dB}$ for a CDC ROADM. As a result, it is no longer necessary to dedicate an amplifier array at the interface between the transit side and add/drop side. In addition, this CDC drop architecture is compatible with transceivers based on direct detection, since the WSSs can carry out the necessary filtering function before the signals reaching the transceiver. Although this $\mathrm{CDC}$ add/drop approach is traditionally associated with the high cost and large footprint, the stacked WSS design proposed enables it to be implemented with a single module, with minimum increase in cost or footprint.
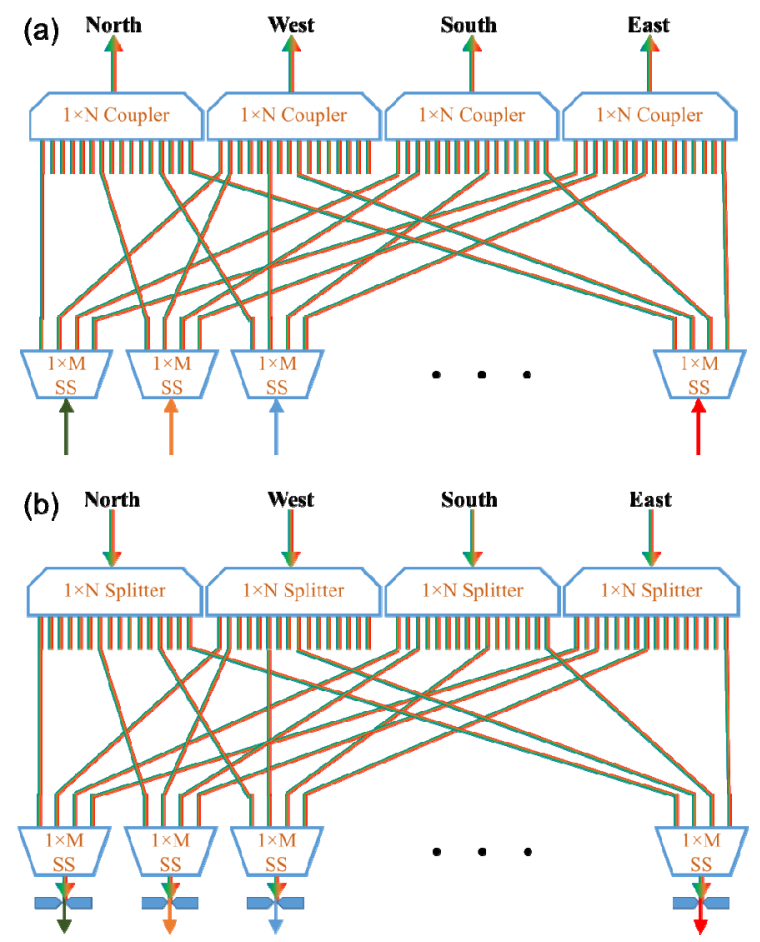

Fig. 5 CDC (a) add and (b) drop structure based on $\mathrm{M} \times \mathrm{N}$ MCSs consisting of an array of $\mathrm{M} 1 \times \mathrm{N}$ splitters/couplers paired with another array of $\mathrm{N} 1 \times \mathrm{M}$ space switches
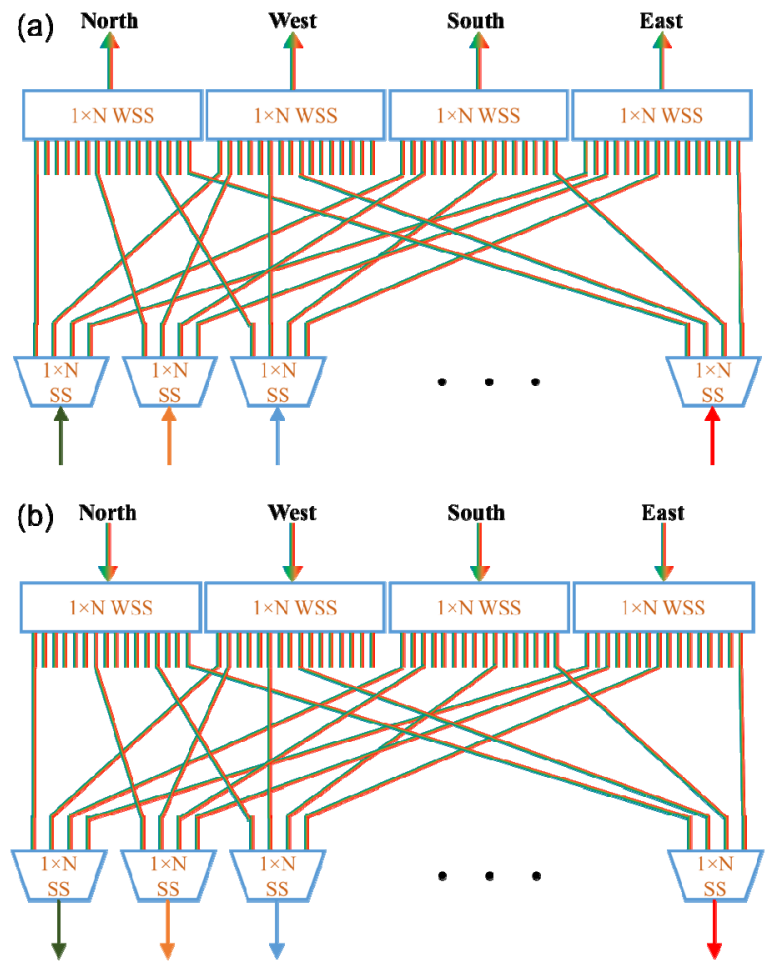

Fig. 6 CDC (a) add and (b) drop structure based on contentionless $\mathrm{M} \times \mathrm{N}$ WXCs consisting of an array of M $1 \times \mathrm{N}$ WSSs paired with another array of $\mathrm{N} 1 \times \mathrm{M}$ space switches 
The stacked WSS module can be further configured as a WXC itself. Fig. 7 illustrates the operational principle of a WXC based on an example of $4 \times 4 \mathrm{WXC}$, in which an array of $41 \times 4 \mathrm{WSS}$ are paired with another array of $41 \times 4 \mathrm{WSSs}$ via a perfect shuffle interconnection. A stacked WSS module in the configuration of $481 \times 12 \mathrm{WSSs}$ can be used to construct 6 independent $4 \times 4 \mathrm{WXCs}, 3$ independent $8 \times 8 \mathrm{WXCs}$, or a single $12 \times 12 \mathrm{WXCs}$. These WXCs could be used as an integrated CDC add/drop module for a ROADM. The $12 \times 12$ WXC configuration is able to interface with a large network node with up to 12 degrees. However, the number of wavelength channels that can be added or dropped is still limited in both cases. To solve this problem, a 'static' CDC architecture was proposed in [28, 31]. In this 'static' CDC

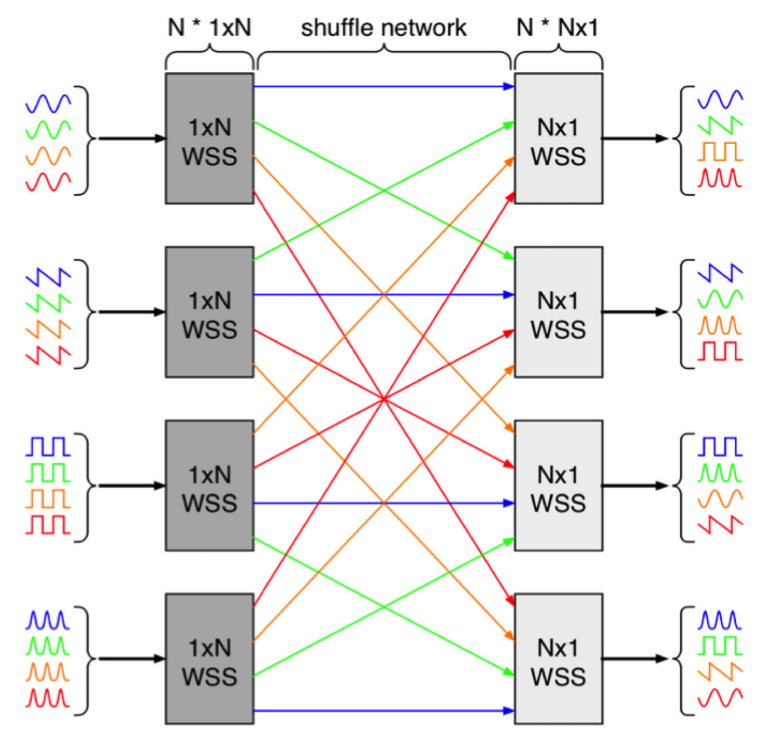

Fig. 7 A contentionless $4 \times 4$ WXCs based on $81 \times 4$ WSSs.
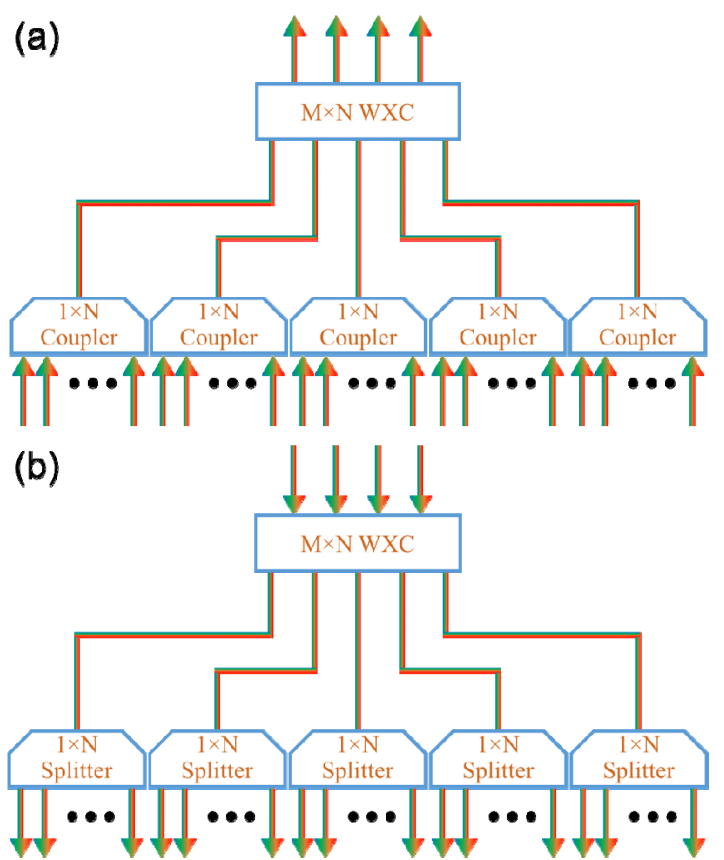

Fig. 8 Static (a) add and (b) drop structures based on contentionless $\mathrm{M} \times \mathrm{N}$ WXC and $1 \times \mathrm{N}$ splitters/couplers. architecture shown in Fig. 8, the WXC is connected to another array of $1 \times N$ splitters/couplers, which increase the number of wavelength channels that could be added or dropped by $N$ times. The $1 \times N$ splitters/couplers used in this 'static' architecture may cause wavelength contention since channels at the same wavelength cannot be added/dropped through a same $1 \times N$ splitters/couplers. However, this wavelength contention can be prevented by the control software, as long as the number of parallel $1 \times N$ splitters/couplers in the array exceed the degree count $(R)$ of the network node. In fact, only $R$ signal channels at a same wavelength can be possibly added or dropped in a $R$-degree network node, since the fibre for each degree cannot carry two signal channels at a same wavelength. Therefore, signal channels at the same wavelength can be assigned to different $1 \times N$ splitters/couplers with minimum impact on the connectivity. Previous studies [28, 31] also suggest the wavelength contention performance in this architecture is comparable to the full CDC solution.

\section{Cost AnALYSis}

This section carries out a cost analysis for the ROADM architectures described in the previous sections. Network nodes of two different sizes are used in this study. The smaller node has 4 degrees, from each of which 4 parallel fibres are entering or exiting, respectively. In the case of the larger node with 8 degrees, the number of parallel fibres is also doubled to 8 . An add/drop ratio of $20 \%$ is assumed in both cases.

The cost of the conventional CDC ROADM based on the standalone WSSs and MCSs is used as the benchmark for comparison. When $4 \times 16 \mathrm{MCSs}$ are used in this architecture, 4 such MCSs are required to add 64 wavelength channels to the 4-degree network node. The same amount of MCSs are required for the drop operation. Correspondingly, $81 \times 7 \mathrm{WSSs}$ are needed at the transit side. For the 7 ports of each WSS, 3 of them are connected to WSSs for other degrees, while the remaining 4 ports are connected to the corresponding $44 \times 16 \mathrm{MCSs}$ at the add or drop side. We could deploy WSSs with a larger port count in this case, and the extra ports will be reserved for future expansion. In order to accommodate the parallel fibres, multiple such ROADMs need to be deployed. It should be noted that signals can only be routed between fibres connected to a same ROADM in this case. For a 4-degree node, $32 \mathrm{WSSs}$ are required at the transit side and $324 \times 16 \mathrm{MCSs}$ are required for the add/drop in total. Considering each $4 \times 16 \mathrm{MCS}$ also requires 4 amplifiers, 128 amplifiers are required. In the case of the 8 -degree node, $8 \times 16 \mathrm{MCSs}$ should be used instead and each of them requires 8 amplifiers to compensate the insertion loss. In total, we need $1288 \times 16 \mathrm{MCSs}, 1024$ amplifiers and $1281 \times 20$ WSSs $(7$ ports for other degrees, 8 ports for MCSs and 5 ports for future expansion.).

The first type of ROADM we are going to analyse is based on a stacked WSS in the configuration of 8 independent $1 \times 32$ WSSs. It will be referred to as 'Architecture I' in this section. At the transit side, each stacked WSS module will be dedicated to the parallel fibres entering or exiting the network node at each degree. For example, the 4 parallel 
fibres entering the 4-degree node from the East will share 4 out of 8 stacked $1 \times 32$ WSSs in a stacked WSS module while the other 4 WSSs within this module are for the exiting fibres. In the case of 8 -degree network node, the 8 parallel fibres can fully exploit the WSSs within one module. As a result, 4 such stacked WSS modules are required for the 4-degree node, and 16 for the 8-degree node. At the add/drop side, the $41 \times 32 \mathrm{WSSs}$ will be paired with $321 \times 4$ space switches to realise a contentionless $4 \times 32 \mathrm{WXC}$, which can be used in the 4-degree node. In total, 16 such WXCs will be needed. These WXCs can be constructed from 8 stacked WSS modules and $5121 \times 4$ space switches. In the case of 8 -degree network node, $81 \times 32 \mathrm{WSSs}$ should be paired with an array of $321 \times 8$ space switches in order to realise an $8 \times 32 \mathrm{WXC}$. Since 64 such $8 \times 32$ WXCs are required to achieve the target add/drop ratio, this architecture will need 64 stacked WSS modules and $20481 \times 8$ space switches. The WXCs in either $4 \times 32$ or $8 \times 32$ configuration are expected to have an insertion loss of $7 \mathrm{~dB}$, which is significantly lower than the $15 \mathrm{~dB}$ insertion loss level in the MCSs. Therefore, the WXCs may no longer need amplifiers to compensate for the loss. Even if the amplifiers are still required, these amplifiers could operate at lower amplification or share the pump laser, which would reduce their unit cost.

The second type of ROADM, whose architecture will be referred to as 'Architecture II' in this section, is based on the 'static' CDC structure described at the end of Section IV. The arrangement of stacked WSS modules at the transit side for both network nodes is kept the same as that in Architecture I. At the add/drop side, the 48 stacked $1 \times 12$ WSS modules are used instead. In the case of 4 -degree node, a module of 48 stacked $1 \times 12$ WSSs can be used to construct 6 independent contentionless $4 \times 4$ WXCs. Such WXC can be subsequently connected to an array of $41 \times 16$ splitters/couplers to realise a $4 \times 64$ WXC unit. This WXC unit is able to add the 64 wavelength channels required for this network node. A second $4 \times 64$ WXC can be constructed in this way using the second $4 \times 4$ contentionless WXCs based on the same stacked WSS module for the drop operation. Each $4 \times 64$ WXC will also need 4 amplifiers. It is also worth mentioning that in this case there are still $44 \times 4 \mathrm{WXCs}$ within this stacked WSS module available for future expansion. In other words, we choose not to use these WXCs for the parallel fibres in order to avoid a potential single point of failure. In total, this 4-degree network node with 4 parallel fibres requires 4 stacked WSS modules, $321 \times 16$ splitters/couplers and 32 amplifiers. In the case of the 8-degree node, the 48 stacked $1 \times 12$ WSS module is used to construct 3 independent contentionless $8 \times 8 \mathrm{WXCs}$, two of which will be used in the conjunction with $1 \times 16$ splitters/couplers for add/drop operations. In total, 8 stacked WSS modules, $1281 \times 16$ splitters/couplers and 128 amplifiers are required for the 'static' CDC add/drop operation.

The cost breakdown for each architecture is calculated and results for the 4-degree and 8-degree network nodes are detailed in the Table II and Table III, respectively. The same information is plotted in Fig. 9 and Fig. 10 to assist the readers' understanding. The cost of each key component is based on the values listed in [27]. They are referenced to a standalone $1 \times 20$ WSS. Our cost analysis for the stacked WSS module detailed in Section II used the same reference point.
The values in the brackets are based on the conservative version of our cost estimation for a stacked WSSs module.

It can be seen from the results for the 4-degree node shown in Table II that both ROADM architectures based on the stacked WSSs module lead to $48 \%$ and $74 \%$ cost savings, respectively, when compared with the benchmark architecture based on the standalone WSSs and MCSs. Even based on the conservative version of our cost estimation, the two proposed ROADM architectures are able to deliver $41 \%$ and $70 \%$ cost reductions. In the transit side, $\sim 80 \%$ cost reduction was realised by using stacked WSSs modules for the parallel fibres in both architectures, with either cost estimation scenarios. At the add/drop site, a modest cost saving of $12 \%$ and a more substantial saving of $67 \%$ were observed in the two proposed architectures, respectively, if the standard cost estimation for the stacked WSSs module is used. The cost saving at the add/drop site of Architecture I is mainly due to the use of low-cost amplifiers, which is made possible by using the more efficient stacked WSSs instead of the passive splitters/couplers. However, the cost saving becomes more marginal if using the conservative version of the cost estimation for the stacked WSSs module. It should be noted that the lower optical losses associated with this architecture will help to reduce the power consumption and therefore the OPEX of the ROADM. Moreover, the add/drop structure used in this architecture has wavelength filtering features, which makes it compatible with low cost transceivers based on the direct detection technology. Meanwhile, Architecture II, with its 'static' add/drop

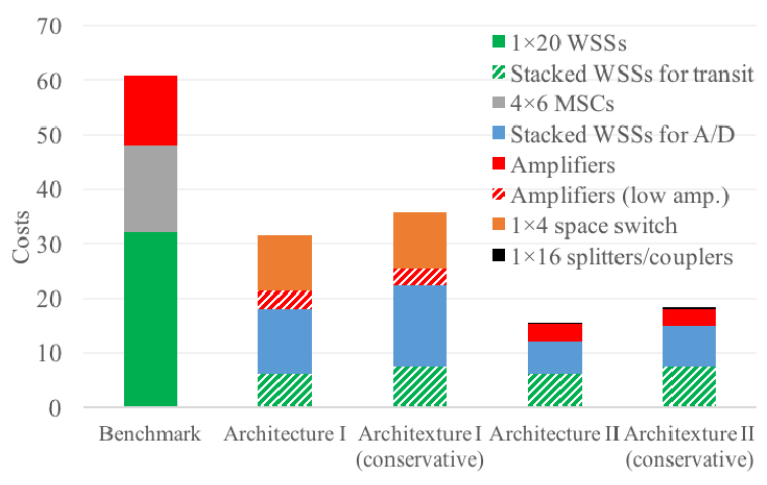

Fig. 9 Cost comparison of ROADM architectures for a 4-degree network node.

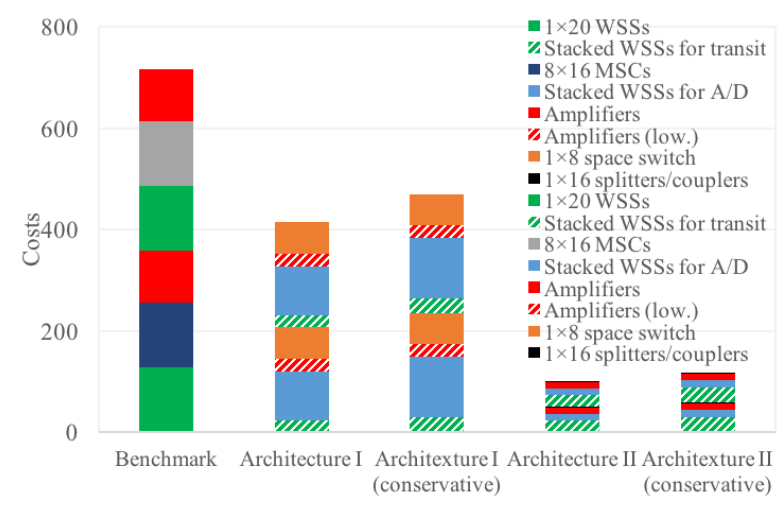

Fig. 10 Cost comparison of ROADM architectures for a 8-degree network node. 
TABLE II

COST COMPARISON OF ROADM ARCHITECTURES FOR A 4-DEGREE NETWORK NODE

\begin{tabular}{|c|c|c|c|c|c|c|c|c|}
\hline & & & Unit cost & Quantity & $\begin{array}{c}\text { Component } \\
\text { cost }\end{array}$ & Transit cost & Add/drop cost & Total cost \\
\hline \multirow{3}{*}{ 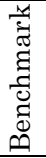 } & Transit & $1 \times 20 \mathrm{WSSs}$ & 1.00 & 32 & 32.00 & \multirow{3}{*}{32.00} & \multirow{3}{*}{28.80} & \multirow{3}{*}{60.80} \\
\hline & \multirow{2}{*}{ Add/drop } & $4 \times 6 \mathrm{MSCs}$ & 0.50 & 32 & 16.00 & & & \\
\hline & & Amplifiers & 0.10 & 128 & 12.80 & & & \\
\hline \multirow{4}{*}{ 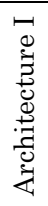 } & Transit & $81 \times 32$ Stacked WSSs Module & $1.50[1.85]$ & 4 & $6.00[7.40]$ & \multirow{4}{*}{$\begin{array}{c}6.00 \\
{[7.40]}\end{array}$} & \multirow{4}{*}{$\begin{array}{c}25.44 \\
{[28.24]}\end{array}$} & \multirow{4}{*}{$\begin{array}{c}31.44 \\
{[35.64]}\end{array}$} \\
\hline & \multirow{3}{*}{ Add/drop } & $81 \times 32$ Stacked WSSs Module & $1.50[1.85]$ & 8 & $12.00[14.80]$ & & & \\
\hline & & $1 \times 4$ space switch & 0.02 & 512 & 10.24 & & & \\
\hline & & Amplifiers (low amplification) & 0.05 & 64 & 3.20 & & & \\
\hline \multirow{4}{*}{ 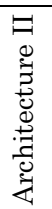 } & Transit & $81 \times 32$ Stacked WSSs Module & $1.50[1.85]$ & 4 & $6.00[7.40]$ & \multirow{4}{*}{$\begin{array}{c}6.00 \\
{[7.40]}\end{array}$} & \multirow{4}{*}{$\begin{array}{c}9.52 \\
{[10.92]}\end{array}$} & \multirow{4}{*}{$\begin{array}{c}15.52 \\
{[18.32]}\end{array}$} \\
\hline & & $481 \times 12$ Stacked WSSs Module & $1.50[1.85]$ & 4 & $6.00[7.40]$ & & & \\
\hline & Add/drop & $1 \times 16$ splitters/couplers & 0.01 & 32 & 0.32 & & & \\
\hline & & Amplifiers & 0.10 & 32 & 3.20 & & & \\
\hline
\end{tabular}

* Values in the brackets are based on the conservative version of the cost estimation in Table I.

TABLE III

COST COMPARISON OF ROADM ARCHITECTURES FOR A 8-DEGREE NETWORK NODE

\begin{tabular}{|c|c|c|c|c|c|c|c|c|}
\hline & & & Unit cost & Quantity & $\begin{array}{c}\text { Component } \\
\text { cost }\end{array}$ & Transit cost & Add/drop cost & Total cost \\
\hline \multirow{2}{*}{ 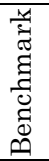 } & \multirow{2}{*}{ Add/drop } & $8 \times 16 \mathrm{MSCs}$ & 1.00 & 128 & 128.00 & \multirow[t]{2}{*}{128.00} & \multirow[t]{2}{*}{230.40} & \multirow[t]{2}{*}{358.40} \\
\hline & & Amplifiers & 0.10 & 1024 & 102.40 & & & \\
\hline \multirow{2}{*}{ 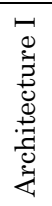 } & Transit & $81 \times 32$ Stacked WSSs Module & $1.50[1.85]$ & 16 & $24.00[29.60]$ & \multirow{2}{*}{$\begin{array}{c}24.00 \\
{[29.60]}\end{array}$} & \multirow{2}{*}{$\begin{array}{c}183.04 \\
{[205.44]}\end{array}$} & \multirow{2}{*}{$\begin{array}{c}207.04 \\
{[235.04]}\end{array}$} \\
\hline & Add/drop & Amplifiers (low amplification) & 0.05 & 512 & 25.60 & & & \\
\hline \multirow{3}{*}{ 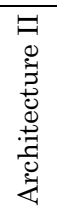 } & Transit & $81 \times 32$ Stacked WSSs Module & $1.50[1.85]$ & 16 & $24.00[29.60]$ & \multirow{3}{*}{$\begin{array}{c}24.00 \\
{[29.60]}\end{array}$} & \multirow{3}{*}{$\begin{array}{c}26.08 \\
{[28.88]}\end{array}$} & \multirow{3}{*}{$\begin{array}{c}50.08 \\
{[58.48]}\end{array}$} \\
\hline & & $481 \times 12$ Stacked WSSs Module & $1.50[1.85]$ & 8 & $12.00[14.80]$ & & & \\
\hline & Add/drop & $1 \times 16$ splitters/couplers & 0.01 & 128 & 1.28 & & & \\
\hline
\end{tabular}

* Values in the brackets are based on the conservative version of the cost estimation in Table I.

features, halves the required number of stacked WSS modules and amplifiers, at the cost of a marginal increase in the contention probability. In addition, the cost reduction is less sensitive to the costs of the stacked WSSs module. Table III shows that the proposed ROADM architectures are able to reduce the cost to a greater extent in the larger 8-degree network node. At the transit side, the architectures based on stacked WSSs modules reduce the cost by $\sim 80 \%$, when compared with the benchmark architecture based on the standalone WSSs with either cost estimation scenarios. At the add/drop side, the cost saving in Architecture $I$ is increased to $21 \%$ or $11 \%$, depending on the versions of the cost estimation for the stacked WSSs module. Again, the cost reduction is primarily driven by the use of low-cost amplifiers, which are enabled by stacked WSSs. Overall, Architecture I is able to deliver a $42 \%$ or $33 \%$ cost reduction, depending on the cost estimation for the stacked WSSs. Architecture II achieves $>85 \%$ cost reduction at the add/drop side in either cost estimation scenarios. The use of $1 \times 16$ splitters/couplers in this architecture substantially reduces the required number of stacked WSSs modules and amplifiers. The total cost of Architecture II is less dependent on the cost of the stacked WSSs module: it is able to realise $\sim 85 \%$ cost reduction in both cases.

\section{CONCLUSION}

This paper presents a stacked WSSs module, in which multiple $1 \times \mathrm{N}$ WSSs can be realised on a single $4 \mathrm{k}$ LCOS device by utilising $2 \mathrm{D}$ beam steering. The stacked module can be designed with a degree of flexibility to either maximise the number of stacked WSSs, e.g. $481 \times 12 \mathrm{WSSs}$, or to realise fewer WSSs with higher port count, e.g. $81 \times 32$ WSSs. The cost of the stacked WSS module is estimated based on its assembly complexity and the component cost. This stacked WSSs module would significantly reduce the cost and the footprint, both of each WSS and each port, due to the extensive sharing of the components and packaging.

The availability of large quantities of low cost WSSs enables the application of multiple WSSs in a ROADM in an economically viable way. Two ROADM architectures based on the stacked WSSs modules are proposed. At the transit 
side of both ROADM architectures, the stacked WSS modules interface the parallel fibres entering or exiting the network node from a same direction, without causing the risk of an additional single point of failure. At the add/drop side of Architecture I, the stacked WSS modules are connected to space switch arrays to construct contentionless WXCs for CDC add/drop. Compared with the benchmark solutions based on MCSs, this design has lower insertion loss and better scalability, and is also compatible with transceivers based on direct detection technology. At the add/drop side of Architecture II, the contentionless WXC is constructed by pairing the WSSs within a single stacked module. The relatively small size of this WXC is addressed by connecting an additional array of splitters/couplers. Although the use of splitters/couplers introduces a small probability of contention, this can be prevented by the wavelength assignment algorithm, while the required the number of stacked WSSs modules and the amplifiers is significantly reduced.

A cost analysis has been carried out for the two proposed ROADM architectures in the 4-degree and 8-degree network nodes, respectively. The results show that Architecture I based on the stacked WSSs brings $~ 40 \%$ cost savings in both network nodes, when compared with the benchmark ROADM architecture based on the MCSs. The cost saving in Architecture I is mainly due to the use of the stacked WSSs module at the transit side of the ROADM. The usage of stacked WSSs modules at the add/drop site can only lead to moderate cost reduction, which is also sensitive to the estimated cost of the stacked WSSs module. However, the stacked WSSs modules will halve the number of required amplifiers, and enable them to operate at lower amplification level. This will leave a positive impact on the OSNR and OPEX.

The cost savings brought by the proposed Architecture II are larger, and it increases with the network node size: a $>70 \%$ cost reduction in a 4 -degree node is increased to $\sim 85 \%$ in an 8-degree node. It should be noted that the splitters/couplers used in the Architecture II potentially cause wavelength contention within individual splitters/couplers. However, this doesn't prevent all the signal channels at a same wavelength to be dropped from or added to the network node simultaneously. In addition, the number of amplifiers at the add/drop side is significantly reduced. This would further reduce the power consumption and the OPEX.

\section{ACKNOWLEDGEMENT}

The authors would like to thank Thierry Zami from Nokia for constructive discussions.

\section{REFERENCES}

[1] M. Sharma, P. Hansen, B. Nayer, and P. Wigley, "Next-generation ROADM technologies and architecture," Proc. SPIE, 2012, vol. 8283, pp. 828309.

[2] B. C. Collings, "Advanced ROADM Technology and Architecture," in Proc. Opt. Fiber Commun. Conf., 2015, Paper Tu3D.3.

[3] T. A. Strasser, and J. L. Wagener, "Wavelength-selective switches for ROADM applications," IEEE J. Sel. Top. Quantum Electron., vol. 51, no. 5, pp. 1150-1157, 2010.
[4] D. M. Marom, D. T. Neilson, D. S. Greywall, P. Chien-Shing, N. R. Basavanhally, V. A. Aksyuk, D. O. Lopez, F. Pardo, M. E. Simon, Y. Low, P. Kolodner, and C. A. Bolle, "Wavelength-selective $1 \times \mathrm{K}$ switches using free-space optics and MEMS micromirrors: theory, design, and implementation," $J$. Lightwave Technol., vol. 23 no. 4, pp. 1620-1630, 2005.

[5] Z. Zhang, Z. You, and D. Chu, "Fundamentals of phase-only liquid crystal on silicon (LCOS) devices," Light-Sci. Appl., vol. 3, pp. e213, 2014.

[6] S. Frisken, G. Baxter, D. Abakoumov, H. Zhou, I. Clarke, and S. Poole, "Flexible and grid-less wavelength selective switch using LCOS technology," in Proc. Opt. Fiber Commun. Conf., 2011, Paper OTuM3.

[7] K. Suzuki, Y. Ikuma, E. Hashimoto, K. Yamaguchi, M. Itoh, and T. Takahashi, "Ultra-High Port Count Wavelength Selective Switch Employing Waveguide-Based I/O Frontend," in Proc. Opt. Fiber Commun. Conf., 2015, paper Tu3A.7.

[8] Y. Ikuma, K. Suzuki, N. Nemoto, E. Hashimoto, O. Moriwaki, and T. Takahashi, " $8 \times 24$ Wavelength Selective Switch for Low-loss Transponder Aggregator," in Proc. Opt. Fiber Commun. Conf., 2015, paper Th5A.4.

[9] M. Iwama, M. Takahashi, Y. Uchida, M. Kimura, R. Kawahara, S. I. Matsushita, and T. Mukaihara, "Low loss $1 \times 93$ wavelength selective switch using PLC-based spot size converter," in Proc. ECOC, 2015, pp. 1-3.

[10] C. Pulikkaseril, L. A. Stewart, M. A. F. Roelens, G. W. Baxter, S. Poole, and S. Frisken, "Spectral modeling of channel band shapes in wavelength selective switches," Opt. Express vol. 19 no. 9, pp. 8458-8469, 2011.

[11] B. Robertson, Z. Zhang, M. M. Redmond, N. Collings, J. Liu, R. Lin, A. M. Jeziorska-Chapman, J. R. Moore, W. A. Crossland, and D. P. Chu, "Use of wavefront encoding in optical interconnects and fiber switches for cross talk mitigation," Appl. Opt., vol. 51 no. 5, pp. 659-668, 2012

[12] B. Robertson, Z. Zhang, H. Yang, M. M. Redmond, N. Collings, J. Liu, R. Lin, A. M. Jeziorska-Chapman, J. R. Moore, W. A. Crossland, and D. P. Chu, "Reduction of crosstalk in a colourless multicasting LCOS-based wavelength selective switch by the application of wavefront encoding," in Proc. SPIE, 2012, vol. 8284, pp. 82840S.

[13] B. Robertson, Z. Zhang, H. Yang, M. M. Redmond, N. Collings, J. Liu, R. Lin, A. M. Jeziorska-Chapman, J. R. Moore, W. A. Crossland, and D. P. Chu, "Application of the fractional Fourier transform to the design of LCOS based optical interconnects and fiber switches," Appl. Opt., vol. 51, no. 12, pp. 2212-2222, 2012.

[14] B. Robertson, H. Yang, M. M. Redmond, N. Collings, J. Liu, A. M. Jerziorska-Chapman, J. R. Moore, Z. Zhang, W. A. Crossland, A. Wonfor, I. H. White, S. H. Lee, and D. Chu, "The Use of Wavefront Encoding to Reduce Crosstalk in a Multicasting Fiber Telecom Switch," in Proc. Opt. Fiber Commun. Conf., 2012, Paper OM2J.6.

[15] H. Yang, B. Robertson, and D. Chu, "Crosstalk reduction in holographic wavelength selective switches based on phase-only LCOS devices," in Proc. Opt. Fiber Commun. Conf., 2014, Paper Th2A.23.

[16] H. Yang, B. Robertson, and D. Chu, "Transient Crosstalk in LCOS Based WSS and a Method to Suppress the Crosstalk Levels," in Proc. Opt. Fiber Commun. Conf., 2013, paper OW1C.3.

[17] H. Yang, B. Robertson, D. Yu, Z. Zhang, and D. P. Chu, "Origin of Transient Crosstalk and Its Reduction in Phase-only LCOS Wavelength Selective Switches," J. Lightwave Technol., vol. 31, no. 23, pp. 3822-3829, 2013.

[18] B. Robertson, H. Yang, M. M. Redmond, N. Collings, J. R. Moore, J. Liu, A. M. Jeziorska-Chapman, M. Pivnenko, S. Lee, A. Wonfor, I. H. White, W. A. Crossland, and D. P. Chu, "Demonstration of multi-casting in a $1 \times 9$ LCOS wavelength welective switch," J. Lightwave Technol., vol. 32, no. 3, pp. 402-410, 2013.

[19] S. Suh, S. Yum, and C. Thevenot, "Fiber-Based Components: Liquid-crystal wavelength selective switches challenge MEMS 
designs”, Available:

http://www.laserfocusworld.com/articles/print/volume-50/issue04/features/fiber-based-components-liquid-crystal-wavelengthselective-switches-challenge-mems-designs.html

[20] W. I. Way, "Optimum Architecture for $\mathrm{M} \times \mathrm{N}$ Multicast Switch-Based Colorless, Directionless, Contentionless, and Flexible-Grid ROADM," in Proc. Opt. Fiber Commun. Conf., 2012, Paper NW3F.5.

[21] V. Kaman, R. J. Helkey and J. E. Bowers, "Multi-Degree ROADM's with Agile Add-Drop Access," in Proc. Photonics in Switching, 2007, pp. 31-32.

[22] L. Zong, G. N. Liu, H. Zhao, T. Ma and A. Lord, "Ultra-compact contentionless ROADM architecture with high resilience based on flexible wavelength router," Proc. Opt. Fiber Commun. Conf., 2014, Paper W2A.64.

[23] L. Zong, H. Zhao, Z. Feng and Y. Yan, " $8 \times 8$ Flexible Wavelength Cross-Connect for CDC ROADM Application," IEEE Photonic Tech. L., vol. 27, no. 24, pp. 2603-2606, 2015.

[24] N. K. Fontaine, R. Ryf and D. T. Neilson, "N×M wavelength selective crossconnect with flexible passbands," in Proc. Opt. Fiber Commun. Conf., 2012, paper PDP5B.2.

[25] H. Yang, B. Robertson, P. Wilkinson, and D. Chu, "Small phase pattern $2 \mathrm{D}$ beam steering and a single LCOS design of $401 \times 12$ stacked wavelength selective switches," Opt. Express, vol. 24, pp. 12240-12253, 2016.

[26] J. R. Moore, N. Collings, W. A. Crossland, A. B. Davey, M. Evans, A. M. Jeziorska, M. Komarcevic, P. J. Parker, T. D. Wilkinson, and H. Xu, "The Silicon Backplane Design for an LCOS Polarization-Insensitive Phase Hologram SLM," IEEE Photonics Tech. L., vol. 20, no. 1, pp. 60-62, 2008.

[27] S. Frisken, "Polarization Diverse Wavelength Selective Switch," U.S. Patent 20150208143 A1, July 19, 2012.

[28] L. Zong, H. Zhao, Z. Feng and Y. Yan, "Low-cost, degree-expandable and contention-free ROADM architecture based on M $\times$ N WSS," in Proc. Opt. Fiber Commun. Conf., 2016, Paper M3E.3.

[29] http://www.lightsmyth.com/

[30] H. Chen, N. K. Fontaine, B. Huang, X. Xiao, R. Ryf and D. T. Neilson, "Wavelength Selective Switch for Dynamic VCSEL-Based Data Centers," ECOC 2016 - Post Deadline Paper; 42nd European Conference on Optical Communication, Dusseldorf, Germany, 2016, pp. 1-3.

[31] S. L. Woodward, M. D. Feuer, P. Palacharla, X. Wang, I. Kim and D. Bihon, "Intra-node contention in a dynamic, colorless, non-directional ROADM," in Proc. Opt. Fiber Commun. Conf., 2010, Paper PDPC.8. 